\title{
Transformation du travail, transformation du métier : quels impacts sur la santé des opérateurs et sur l'activité collective?
}

Transforming work, changing profession: Effects on occupational health Transformación del trabajo, transformación del oficio: impacto sobre la salud de los operadores y sobre la actividad colectiva?

Lucie Cuvelier et Sandrine Caroly

\section{OpenEdition}

Édition électronique

URL : http://journals.openedition.org/pistes/1732

DOI : $10.4000 /$ pistes. 1732

ISSN : 1481-9384

Éditeur

Les Amis de PISTES

Édition imprimée

Date de publication : 1 mai 2011

\section{Référence électronique}

Lucie Cuvelier et Sandrine Caroly, «Transformation du travail, transformation du métier : quels impacts sur la santé des opérateurs et sur l'activité collective? », Perspectives interdisciplinaires sur le travail et la santé [En ligne], 13-1 | 2011, mis en ligne le 01 mai 2011, consulté le 30 avril 2019. URL : http://journals.openedition.org/pistes/1732 ; DOI : 10.4000/pistes.1732

Ce document a été généré automatiquement le 30 avril 2019.

\section{cc) (†)}

Pistes est mis à disposition selon les termes de la licence Creative Commons Attribution - Pas d'Utilisation Commerciale - Pas de Modification 4.0 International. 


\title{
Transformation du travail, transformation du métier : quels impacts sur la santé des opérateurs et sur l'activité collective?
}

\author{
Transforming work, changing profession: Effects on occupational health \\ Transformación del trabajo, transformación del oficio: impacto sobre la salud de \\ los operadores y sobre la actividad colectiva?
}

Lucie Cuvelier et Sandrine Caroly

\section{Introduction}

1 Avec le processus d'ouverture à la concurrence engagé depuis le début des années 1990, les services postaux européens se modernisent en profondeur : réorganisation du réseau de traitement du courrier, délocalisation et construction de nouvelles plates-formes de tri en dehors des centres-villes, automatisation des chaînes de production, etc. Ces mutations, qui nécessitent que les opérateurs développent de nouvelles compétences et reconstruisent leur activité, sont susceptibles d'avoir des répercussions sur leur santé. L'objectif de cette étude est de caractériser l'impact de tels changements organisationnels et techniques sur la santé et sur l'activité collective des opérateurs.

2 Les travaux sur le thème du changement proviennent de disciplines très variées: management, gestion, psychologie du travail, sociologie, économie, et bien d'autres champs encore. Le caractère touffu et diversifié de cette littérature fait écho à l'« ambiguïté » et à la « banalité » du terme « changement », et aux multiples facettes sous lesquelles il peut être abordé (Bernoux, 2004 ; Guibert, 2009). En ergonomie, les études peuvent se positionner en aval ou en amont de ces processus de transformation. L'ergonome intervient souvent a posteriori, à la demande des entreprises, pour évaluer « après coup » les conséquences des changements apportés aux différentes dimensions de 
l'activité et sur la santé des opérateurs (Pavageau, Nascimento et Falzon, 2006 ; BobillierChaumon et Dubois, 2007). Il peut aussi intervenir en amont, et prendre part à la conception des systèmes de travail: c'est d'ailleurs parfois lui qui impulse des changements au travers des recommandations qu'il formule (Lamonde, 2004 ; Daniellou, 2004 ; Béguin, 2004). Mais les études ergonomiques sont rarement focalisées sur l'impact des processus de changements sur la santé des travailleurs et sur l'activité collective. Car « la myopie » de l'ergonome (Darses et de Montmollin, 2006) ne l'incite pas à aborder cette notion de "changement» dans sa globalité. En regroupant les contraintes extérieures à l'entreprise, les modifications des organisations et des techniques, les décisions et les représentations de multiples acteurs, le terme "changement » apparaît effectivement comme un terme «flou [qui] ne permet pas de cerner avec précision l'objet que l'on veut observer » (Bernoux, 2004, p. 7). Certains auteurs déplorent alors le fait qu'il n'existe que peu de données nous renseignant sur les changements rencontrés concrètement par les travailleurs dans leur environnement de travail (Zanet et coll., 2004). Pourtant «l'analyse ergonomique des changements organisationnels est une nécessité et un enjeu pour l'ergonomie qui ne dispose que de peu d'outils pour cela. Des modèles sociologiques ou organisationnels peuvent nous aider à comprendre cette dynamique et la manière dont elle fragilise l'opérateur en activité » (Valot, 2001, p. 24). Mais, d'après cet auteur, il est désormais nécessaire de s'orienter vers une " compréhension à finalité ergonomique du changement ».

\section{Contexte de l'intervention}

\subsection{Le métier de facteur}

Dernier maillon de la chaîne de distribution du courrier, les facteurs apportent six jours sur sept les lettres et les colis au domicile des particuliers et des entreprises. Réalisée à pied, à bicyclette, en cyclomoteur ou en automobile, cette «tournée du facteur ${ }^{1}$ » ne représente en fait qu'environ la moitié de leur temps de travail. À leur arrivée au centre de distribution à $6 \mathrm{~h} 40$, les facteurs débutent leur journée par le tri général dit «TG3». Durant presque une heure, les lettres et la presse sont réparties par « quartier » c'est-àdire par tournée de facteur sur des casiers spécifiques au TG3 (sur ces casiers, tous identiques, chaque case correspond à une tournée). Puis après avoir « décasé » (i.e. relevé le courrier de son secteur sur chacun des casiers du TG3), chaque facteur rejoint sa " position » où il va pouvoir débuter le tri de sa tournée sur un casier qui lui est propre (TG4). Sur certaines tournées (à pied, en vélo ou en cyclomoteur) les plis, une fois triés, doivent être « bottés » c'est-à-dire rassemblés en liasses maintenues par une ficelle ou des élastiques avant d'être chargés dans les sacoches. Les heures de départ « en distribution " s'échelonnent entre $9 \mathrm{~h} 30$ et $10 \mathrm{~h}$. À leur retour au centre, les facteurs « rendent les comptes » (objets spéciaux non distribués, « contre-remboursements »...) et traitent « les rebus » (destinataire n'habitant pas à l'adresse indiquée, nom non inscrit sur la boîte à

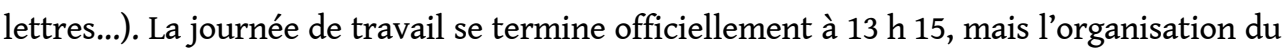
travail étant basée sur le "fini-parti », elle peut se terminer avant ou après l'heure prévue. 


\subsection{Le changement suivi}

4 L'originalité de cette "recherche-action », menée dans un centre de tri et de distribution du courrier, est d'être basée sur le suivi d'un changement organisationnel et technique majeur. Du jour au lendemain, une équipe de 37 facteurs et son encadrement ont déménagé dans de nouveaux locaux et ont dû s'adapter à l'implantation d'une machine de tri et de nouveaux outils (en particulier des nouveaux casiers de tri du courrier). Ces évolutions techniques ont été accompagnées de plusieurs réorganisations du travail. En effet, la mécanisation du courrier a deux principales conséquences :

- une diminution du temps de tri au profit du temps de distribution, puisque désormais, seules les lettres de format «non standard» (lettres trop petites ou trop grandes, presse, lettres non lues par les machines...) continuent d'être triées à la main ;

- la suppression de trois tournées : les secteurs de ces tournées ont été répartis sur les 25 tournées restantes dont les parcours ont été rallongés (ceci engendrant d'autres répercussions sur l'activité de travail telle l'évolution des moyens de locomotion: passage du vélo au scooter par exemple).

5 Ces transformations concernent tellement d'aspects à la fois - espaces, organisations, valeurs et sens du métier, dispositifs techniques - qu'elles instaurent une véritable " coupure ", une bascule vers un nouveau système (Lacoste, 1992).

Dans ce contexte, la demande à laquelle nous avons répondu était portée par la médecine de prévention professionnelle qui s'interrogeait sur les liens potentiels entre les changements effectués (en particulier l'introduction d'un nouveau casier de tri) et la santé des facteurs. La problématique des troubles musculo-squelettiques (TMS), pathologie prépondérante dans l'entreprise, fut plus particulièrement pointée. La phase de conception du changement et des dispositifs techniques, réalisée au niveau national de l'entreprise, n'a pas pu être observée. Seule l'activité de travail sur le centre de tri, avant, pendant et après la mise en œuvre du changement a été analysée. L'analyse de la demande auprès des différents acteurs a conduit à reformuler les objectifs de l'intervention de la façon suivante: cette étude vise à caractériser l'impact du changement sur la santé des opérateurs, la santé étant à définir "au sens large " physique, cognitive, psychologique et sociale - en incluant le sens et la valeur du métier, afin d'en dégager une réflexion sur la conduite des projets futurs. En effet, le site où nous avons mené cette étude était un site pilote permettant d'élargir la démarche de changement à d'autres sites. Cette approche globale de la santé tient en particulier compte des dimensions collectives de l'activité (cf. État de l'art). Ainsi, l'évolution des contraintes biomécaniques et organisationnelles sera décrite et les répercussions de cette évolution sur la santé et sur l'activité réelle (activité collective notamment) seront examinées.

\section{3 État de l'art}

\subsection{Les TMS : une atteinte globale de la santé des travailleurs}

7 Maladies professionnelles reconnues les plus répandues en France, les troubles musculosquelettiques (TMS) apparaissent aujourd'hui comme des pathologies «tenaces » et «résistantes à la prévention» (Bourgeois et coll., 2006). L'approche biomédicale 
classique, qui aborde les TMS par la compréhension des sollicitations musculosquelettiques, est une voie de prévention incontournable pour laquelle il faut encore « encourager le développement des connaissances en physiologie et en biomécanique [et également en] épidémiologie " (Vézina, 2001, p. 53). Mais les limites d'un modèle de prévention basé exclusivement sur une approche biomécanique du geste font aujourd'hui consensus : "Il est maintenant légitime de penser que la caractérisation, d'un point de vue uniquement physique [force, répétitivité, posture de travail], de la sollicitation biomécanique est insuffisante pour expliquer la genèse d'un TMS » (Aptel et Hubault, 2005, p. 18). Dans le domaine biomédical, les résultats les plus récents insistent sur l'intérêt de prendre en compte les facteurs psychosociaux et le stress dans les études épidémiologiques et biologiques (Aptel et Caudez, 2005). Par ailleurs, la plupart des études réalisées en ergonomie montrent que «le phénomène se joue sur une estrade plus large, [...] où la situation conjugue des dimensions multiples, qui balaient un spectre qui va du psychique au gestionnaire » (Hubault, 2005).

8 Certains champs relevant davantage des sciences humaines, comme la psychologie, la psychodynamique ou la philosophie, nous permettent d'ouvrir notre regard sur les TMS : ils envisagent cette pathologie comme une atteinte à la santé «dans toutes ses dimensions ", " une dimension parmi d'autres de la vie humaine et de la vie au travail » (Coutarel, 2004, p.111). Selon ces approches, la santé se manifeste à travers les possibilités de développer ses compétences, les moyens collectivement construits du pouvoir d'agir au travail. Elle est fortement reliée à la perception qu'a chaque individu "d'être pour quelque chose dans ce qui lui arrive ». On peut par exemple évoquer, pour illustrer ces approches, les notions de gestes « empêchés » ou " contrariés » développées par Clot (Clot, 2004 ; Clot et Fernandez, 2005). Selon cet auteur, les TMS peuvent être expliqués par un "sous-développement» des mouvements lié à une «hyposollicitation des activités d'appropriation du milieu de travail par les sujets » (Clot, 2005) : l'opérateur compose alors avec une gamme de gestes et d'automatismes réduite. Ceci peut engendrer des « passages en forces » ayant comme effet une surcharge biomécanique (Bourgeois et coll., 2006).

9 L'approche des ergonomes s'inscrit dans cette approche globale de la santé: une intervention en ergonomie ne vise pas seulement à pallier une déficience ou à prévenir la dégradation de la santé, mais aussi à favoriser la construction de la santé, à travers le développement d'une approche active (Falzon, 1996). Il s'agit certes d'éviter les diverses formes de déclin de la santé au travail, mais aussi de "prêter attention à la maitrise des déficiences (par des stratégies de compensation), à l'accroissement et à la valorisation de l'expérience (par la maturation des évènements et l'utilisation des compétences), et à la réalisation d'un rôle social, avec sa reconnaissance économique » (Laville et Volkoff, 1993). Pour cela, les ergonomes s'appuient notamment sur le concept de «marge de manœuvre » qui « établit un lien entre la santé et le contrôle de chacun sur sa propre situation de travail» (Coutarel, 2004, p. 172). Les relations que les individus établissent entre eux au sein des collectifs sont alors des ressources pour ouvrir ces marges de manœuvre.

\subsection{L'activité collective : une ressource pour la santé des travailleurs}

10 Plusieurs recherches (Assunçao, 1998; De la Garza et Weill-Fassina, 2000 ; Barthe, 2000) révèlent « le rôle central des activités collectives dans les systèmes sociotechniques, tant 
sur le plan de la performance que sur celui de la santé, de la sécurité, de la fiabilité » (Flageul-Caroly, 2001; p. 123). De façon générale, il y a "activité collective » lorsque les opérateurs sont mutuellement dépendants les uns des autres (Leplat, 1993; Schmidt, 1991). Cette notion d'«activité collective» rassemble en fait diverses formes d'interactions sociales. De la Garza et Weill-Fassina (2000) distinguent ainsi plusieurs modalités d'interactions permettant de caractériser le travail collectif. Parmi celles-ci, sont décrites notamment la coopération, la collaboration et l'entraide. La collaboration concerne "une activité collective dans laquelle les opérateurs accomplissent sur un même objet des opérations différentes qui s'articulent les unes aux autres avec un but commun à court ou moyen terme " (De la garza et Weill-Fassina, 2000, p. 228). Elle implique différentes unités de travail ne partageant pas obligatoirement le même espace géographique. La coopération se distingue de la collaboration par une dimension spatiotemporelle beaucoup plus réduite. En effet, la coopération est une situation dans laquelle "les opérateurs travaillent ensemble sur le même objet ou un objet proche visant au même but proximal » (De la Garza et Weill-Fassina, 2000, p. 228). Elle implique un partage des tâches entre les acteurs en fonction des connaissances et compétences de chacun et selon les exigences immédiates du travail, l'état de santé des uns et des autres ou la force physique présente. Pour Hoc (2001) on ne peut parler de coopération que si les deux conditions suivantes sont réunies :

- les opérateurs poursuivent chacun des buts qui peuvent entrer en interférence (sur le plan des résultats ou des procédures);

- les opérateurs font en sorte de traiter ces interférences afin de faciliter les activités des uns et des autres.

11 Enfin, l'aide et l'entraide sont sous-tendues par des notions d'échanges et visent «bien sûr à répondre aux objectifs de la production, mais [leur] mobile essentiel est d'ordre social » (Assunçao, 1998, p. 63). Il s'agit, « selon les cas soit de seconder quelqu'un dans ses fonctions" soit d'" exécuter une action ou une opération à sa place » (op. cit.). Ces modalités de réalisation du travail collectif ne s'excluent pas les unes des autres: elles peuvent concerner différents groupes d'acteurs dans l'entreprise et sont susceptibles d'apparaître successivement, voire simultanément en fonction du déroulement de l'activité et des différentes phases du travail (De la Garza et Weill-Fassina, 2000).

La notion de "collectif de travail» se distingue de ces diverses formes d'interactions collectives car elles relèvent de conditions supplémentaires: "Tout travail collectif n'implique pas un collectif de travail» (Weill-Fassina et Benchekroun, 2000, p. 6). Même si, «la notion est encore loin d'être stabilisée en ergonomie » (Flageul-Caroly, 2001, p. 123), les conditions d'élaboration du «collectif de travail » proposées par Cru (1988) font désormais consensus au sein de la communauté (Dessors, Dejours et Molinier, 1994 ; Clot ; 2004, Caroly, 2010 ; Weill Fassina et Benchekroun, 2000). Pour pouvoir parler de collectif de travail, «il faut simultanément plusieurs travailleurs, une œuvre commune, un langage commun, des règles de métier, un respect durable de la règle par chacun, ce qui suppose un cheminement individuel qui va de la connaissance des règles à leur intériorisation" (Cru, 1988). Ces règles de métier sont à différencier à la fois des règlements, procédures, normes ou consignes applicables sur les lieux de travail et du référentiel opératif commun nécessaire à la coopération. Elles constituent un système de valeurs de référence qui ne peut être réduit aux dimensions techniques du travail. Ces valeurs définissent «le métier» (Cru, 1995). Elles s'inscrivent dans une tradition 
historique, avec des savoir-faire qui précisent les attitudes à tenir notamment lorsque la situation n'est pas totalement définie par les règles (Daniellou, Simard et Boissières, 2009). Cette notion de métier peut être rapprochée de la notion "genre professionnel » qui désigne la manière dont les opérateurs définissent leur rapport aux objets et aux autres, ce qu'ils s'autorisent à faire dans les relations sociales, et ce qu'ils s'empêchent de faire (Clot, 2004).

En gérant ainsi la diversité des styles personnels dans les gestes à réaliser, les collectifs professionnels sont, au moins pour partie, gardiens des « règles du métier ». En ce sens, ils apparaissent favorables à la prévention des TMS et à la construction de la santé au sens large (Coutarel, 2004). D'une part, car ils protègent les individus: «Le sentiment de communauté constitue une défense très efficace vis-à-vis des attaques extérieures " (Davezies, 2005, p. 25). Plusieurs études montrent ainsi que, d'une façon générale, «la santé se dégrade en milieu de travail lorsqu'un collectif professionnel devient une collection d'individus exposés isolément » (Caroly et Clot, 2004, p. 43 ; Clot, 2010). Ceci est en particulier observé dans les études sur la prévention des TMS: le partage des connaissances, les régulations de l'activité, le sens du travail sont des notions fortement liées aux modalités de réalisation collective du travail qui peuvent expliquer pour partie l'occurrence de cette pathologie (Bourgeois et coll., 2006). D'autre part, les collectifs professionnels sont des ressources pour le développement des compétences. Ils contribuent, par exemple, à la capitalisation de l'expérience lors de «l'accueil et [de] la formation des nouveaux, auxquels ils peuvent transmettre des formes de connaissances distinctes de celles enseignées par l'entreprise » (Daniellou, Simard et Boissières, 2009; Daniellou et coll., 2008). Ils permettent en outre la construction de compétences à travers des régulations collectives et les entraides mises en place pour faire face aux variations d'état de chacun (Gaudart et Weill Fassina, 1999; Gaudart et Pueyo, 2000 ; Pueyo, 1999). «Le collectif permet au salarié d'apprendre des règles mais également de s'économiser, se protéger ou progresser en confrontant ses propres savoirs et stratégies à ceux de ses collègues» (Pavageau, Nascimento et Falzon, 2007). Ainsi, la production et le maintien d'un collectif de travail comporte de multiples enjeux : permettre aux individus de ne pas être isolés, pouvoir créer des zones d'action dans et en dehors des marges de manœuvre potentielles, donner des références et des moyens de réélaborer les règles pour diminuer les conflits de but, etc. (Caroly et Clot, 2004). Le collectif de travail, en tant que «garant du métier » est donc une ressource essentielle pour la santé au travail.

\section{Méthode}

Plusieurs méthodes ont été mises en œuvre avec pour objectif, d'une part, de mettre en discussion l'évolution des contraintes et des conditions de réalisation du travail au cours du changement et, d'autre part, d'en prévoir les conséquences sur la santé des opérateurs et les formes de réalisation collective du travail. L'analyse développée est essentiellement qualitative. Des observations ouvertes de l'activité des 37 facteurs du site ont été menées avant et après le changement, durant les phases de tri et de distribution du courrier. Des observations systématiques ( 45 heures au total) des activités de tri et de distribution ont aussi été conduites auprès de 15 opérateurs (cf. tableau 1).

Cette analyse est complétée par des éléments quantitatifs, avec la diffusion d'un questionnaire. Le questionnaire choisi (Roquelaure et coll., 2001; Roquelaure et coll., 2002) fut celui utilisé au sein du groupe de recherche sur la prévention durable des TMS 
dans lequel nous nous inscrivions (Daniellou et coll., 2008). Il a fait l'objet de deux passations : l'une deux semaines avant le changement et l'autre, quatre mois et demi après le changement. La détermination de ces deux moments de passation fut délicate (cf. discussion). En outre, cette double passation ne vise pas une analyse épidémiologique des TMS, car ces pathologies peuvent se déclarer après de longues durées d'exposition : elles doivent donc faire l'objet d'un suivi systématique et régulier sur du long terme (Aptel, Cail et Aublet-Cuvelier, 2009 ; Lasfargues, Roquelaure, Fouquet et Leclerc, 2003). Mais les réponses aux questionnaires permettent de dresser « deux photographies » de l'état de santé des opérateurs sur le site, avant et après changement, dans le but d'une comparaison quantitative, complémentaire aux analyses qualitatives. Vingt (en début de phrase, les nombres doivent être écrits en lettres) personnes ont répondu lors de la première passation, 23 lors de la seconde et, parmi ces répondants, 15 ont participé aux deux passations. Une première analyse avait pour but de considérer les caractéristiques sociodémographiques (genre, âge, statut, ancienneté, horaires de travail...) et l'état de santé générale de la population à ces deux moments. Cette analyse montre que les caractéristiques des répondants reflètent la diversité des caractéristiques de la population sur le site (cf. tableau 1). L'analyse a alors porté sur l'évolution des douleurs ressenties (en particulier leur localisation) et des contraintes organisationnelles et biomécaniques perçues sur l'échantillon de 15 personnes, commun aux deux moments de passation.

Tableau 1. Caractéristiques des sujets impliqués aux différentes étapes de l'étude (effectifs selon le genre et l'âge des individus)

\begin{tabular}{|l|l|l|l|l|l|l|}
\hline & \multicolumn{2}{|l|}{ Genre } & \multicolumn{2}{l|}{ Âges (en années) } & \\
\hline & Femmes & Hommes & $<35$ & {$[35.45]$} & $<45$ & Total \\
\hline Observations systématiques & 8 & 7 & 5 & 6 & 4 & 15 \\
\hline Questionnaire (échantillon commun) & 7 & 8 & 3 & 4 & 8 & $\mathbf{1 5}$ \\
\hline Entretien collectif (facteurs invités) & 4 & 4 & 2 & 3 & 3 & $\mathbf{8}$ \\
\hline
\end{tabular}

En parallèle, des entretiens individuels " informels » et deux entretiens collectifs ont été conduits afin de mieux comprendre les motifs de l'activité et les logiques qui la soustendent. Les participants aux entretiens collectifs (huit facteurs «invités») ont été choisis en fonction des observations réalisées en amont et selon des critères d'âge et de sexe (cf. tableau 1.). Ces participants n'ont pas nécessairement répondu aux questionnaires, ces derniers ayant été « anonymisés " par le médecin de prévention avant nos analyses. Finalement, sept puis six facteurs se sont présentés. Il s'agissait des mêmes opérateurs lors de ces deux réunions. La première était un entretien collectif de coanalyse des premiers résultats. Son objectif était d'observer comment, à travers les échanges dans l'équipe, les contraintes liées au changement avaient été intégrées et s'inséraient dans une dimension collective du travail. C'est donc le thème de «l'activité de travail dans le changement » qui fut au cœur de cet entretien collectif. Les modifications relevées par les chercheurs ont été présentées par thèmes successifs, sous la forme d'une comparaison avant/après (localisation, locaux, machines, outils, gestes, tournées, véhicules, 
organisation du travail), et le groupe a débattu de l'impact de chacune de ces modifications sur l'activité de travail. Le second entretien fut un entretien de "confrontation collective", basée sur les techniques d'autoconfrontation et d'alloconfrontation (Mollo et Falzon, 2004). L'autoconfrontation consiste à faire verbaliser un sujet à partir de traces de sa propre activité, tandis que l'allo-confrontation consiste à faire verbaliser un sujet à partir de traces de l'activité d'un collègue. Lorsqu'il s'agit d'une " confrontation collective", c'est un groupe de participants qui verbalisent au sujet de l'activité de l'un ou de plusieurs d'entre eux (Mollo, 2004). Ce type d'entretien favorise l'activité réflexive : cela permet aux participants de prendre conscience d'autres formes de connaissances, d'évaluer et de justifier les différents modes opératoires possibles. Ces confrontations donnent ainsi des possibilités de construction de nouvelles connaissances. Dans notre étude, trois opérateurs volontaires ayant développé des stratégies différentes pour trier le courrier sur les nouveaux casiers ont été filmés. Un montage vidéo a permis de réaliser cinq minutes de film par personne à partir d'extraits sélectionnés. L'entretien collectif (six participants) s'est ensuite déroulé comme suit. Pour chaque film, la personne filmée commentait la vidéo de son activité (autoconfrontation) en ayant la possibilité de l'arrêter à la demande. Puis, le groupe réagissait pendant dix minutes sur le film et les propos du facteur filmé (allo-confrontation collective). Enfin, le facteur filmé terminait la séquence en concluant sur l'échange qui avait eu lieu entre ses collègues. Les deux entretiens collectifs (une heure trente et deux heures chacun) ont été enregistrés puis retranscrits intégralement. Les verbalisations ont ensuite fait l'objet d'une analyse de contenu fondée sur un traitement thématique.

Enfin, la tenue d'un journal de bord dans le cadre du projet sur «la prévention durable des TMS » (Daniellou et coll., 2008; Landry, 2008) s'est révélée un outil très utile dans le suivi et l'analyse d'un changement. Relevant à la fois «du journal d'exploration, du journal intime et du dossier clinique» (Bellemare, Marier, Allard, 2001), il a permis de formaliser par écrit au fur et à mesure les impressions et réflexions exprimées « à chaud » et de consigner les évolutions du contexte.

\section{Résultats}

\subsection{Le changement et la santé}

\subsection{1 « Le métier de facteur, un métier pénible » : état des lieux avant changement}

Tous les acteurs rencontrés s'accordent à dire que le métier de facteur est un métier « physique », " difficile ». La distribution, même si elle peut être vécue comme un moment de libération (Clot et coll., 2000) par rapport aux travaux intérieurs, voire un gage d'indépendance et de liberté (Dubar et coll., 2001), reste la phase de travail décrite comme la plus «pénible ». Les tournées " deux roues » sont communément jugées plus fatigantes. Les facteurs y sont en effet fortement exposés aux intempéries qui, en plus de créer des inconforts (froid, humidité, chaleur...), augmentent les contraintes de travail : les lettres ne doivent pas être mouillées, les plis s'envolent par fort vent, la neige et le verglas empêchent l'accès à certaines voies ou givrent les boîtes aux lettres, etc. Que ce soit en vélo ou en scooter, le démarrage, surtout lorsque l'engin est très chargé, nécessite un effort important.

«Les scooters sont lourds, ils doivent être posés très souvent; et pour repartir, il

est nécessaire de les soulever et de donner un coup de rein ». 

départs sur les trois heures quinze de distribution, alors que la factrice disait éviter au maximum le nombre d'arrêts, préférant se déplacer à pied plutôt que d'avoir à déplacer l'engin (250 déplacements à pied). Les tournées en voiture échappent pour partie à ces pénibilités. Néanmoins, elles nécessitent des ports de charge et des postures contraintes par l'habitacle, notamment lors des montées et descentes du véhicule. Sur les 3 observations systématiques de tournées en voiture réalisées, nous avons compté entre 150 et 300 sorties du véhicule (montées et descentes). Toutes ces contraintes génèrent

" des douleurs qu'on ne ressent pas forcément à chaud mais qui se déclenchent, une

fois chez soi, au repos. »

traux de tri sont soumis à une forte standardisation : les cadences utilisées par les organisateurs vont de 800 à 2500 lettres triées à l'heure, soit entre 13 et 42 lettres manipulées par minute. Même si le «facteur peut prendre le temps qu'il souhaite pour préparer sa tournée », le contexte du "fini-parti » fait que bien souvent ces cadences sont suivies. Cette phase de tri implique donc la réalisation de gestes répétitifs des membres supérieurs (généralement membre droit) à une fréquence élevée durant presque trois heures par jour. Du fait de la hauteur des casiers, l'épaule est fréquemment sollicitée. La prise des lettres et leur dépôt dans les cases requièrent en outre des mouvements fréquents du coude et du poignet.

\subsubsection{Analyse comparative avant/après le changement : résultats quantitatifs du questionnaire}

D'une façon générale, un « questionnaire se fonde sur le point de vue de l'opérateur donc, entre autres, sur sa perception des effets du travail sur sa santé» (Volkoff, 2005, p. 29). L'utilisation de cette technique permet ainsi de considérer le vécu des opérateurs, «indicateur particulier mais essentiel», tout en réduisant «les effets paradoxaux de l'expression ou de la non-expression de la parole » (Bourgeois et coll., 2006, p. 212). Mais l'analyse de ce type de résultat est délicate et les interprétations doivent être faites avec prudence. Les limites de cette méthode quantitative sont notamment liées aux modalités de passation et au faible échantillon de répondants sélectionnés sur la base du volontariat (cf. ( ?) Discussion). En outre,

« l'utilité d'un questionnaire est étroitement conditionnée à son intégration dans un processus dont l'observation de l'activité in situ [...] reste la clef de voûte fondamentale » (Prunier-Poulmaire et Gadbois, 2005, p. 85).

Néanmoins, malgré ces limites, la double passation du même questionnaire avant et après le changement permet de constater une évolution qualitative et quantitative des douleurs perçues par les opérateurs. On observe notamment qu'au sein de l'échantillon commun aux deux passations (15 personnes), le nombre d'opérateurs déclarant avoir ressenti des douleurs, courbatures, gênes ou engourdissements au cours des sept derniers jours est resté stable, mais que le nombre total de douleurs déclarées a augmenté (cf. figures 1 et 2). Une analyse plus fine révèle que ce sont les personnes qui ont déclaré des douleurs avant le changement qui en déclarent davantage après. On constate aussi que les localisations de ces douleurs ne sont pas les mêmes: trois mois après le changement, davantage d'opérateurs souffrent des membres supérieurs (épaules ou bras) et de la nuque, tandis qu'ils sont moins nombreux à signaler des douleurs au niveau de la main ou du poignet ou bien du bas du dos (cf. figure 3). Ainsi, même si le nombre global de personnes déclarant des douleurs est constant, le changement semble avoir fragilisé la

Perspectives interdisciplinaires sur le travail et la santé, 13-1 | 2011 
santé des opérateurs, et plus particulièrement celle des opérateurs déjà atteints avant le changement.

Figure 1. Nombre de personnes ayant perçu au moins une douleur au cours des sept derniers jours

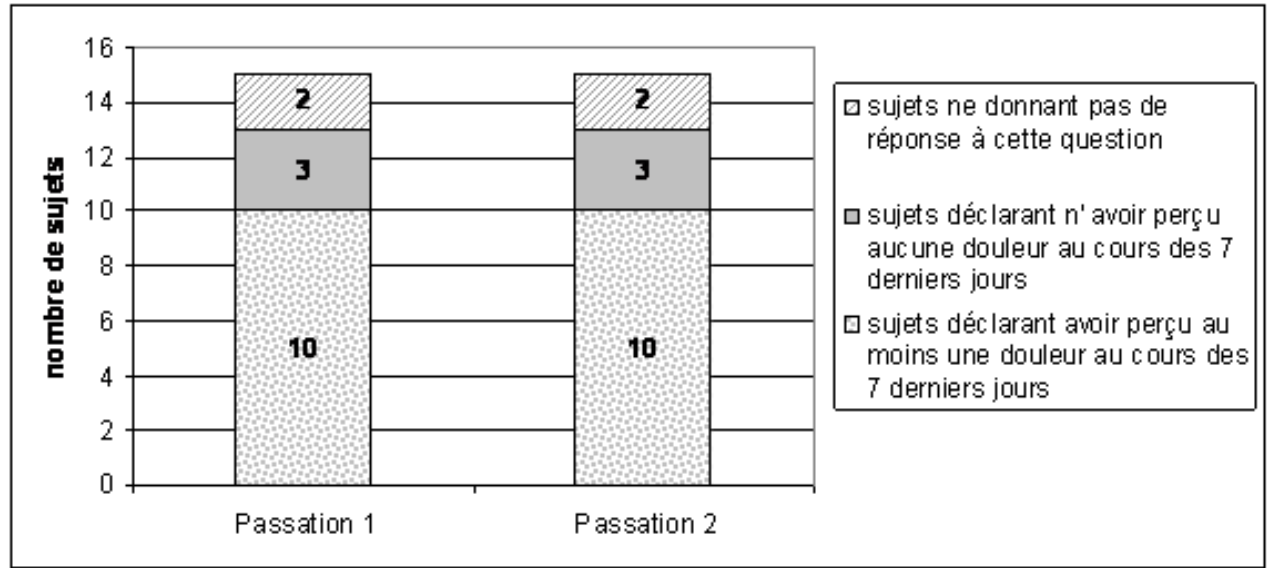

Comparaison des résultats issus de la passation avant changement (passation 1) et de la passation après le changement (passation 2) sur l'échantillon commun aux deux passations ( $n=15$ sujets)

Figure 2. Nombre total de douleurs perçues au cours des sept derniers jours

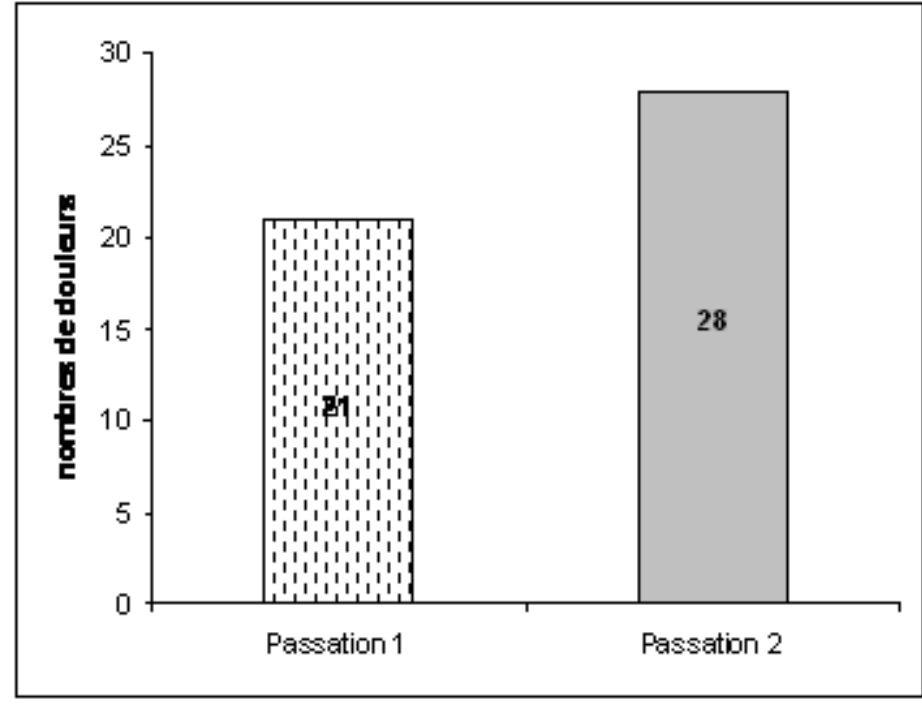

Comparaison des résultats issus de la passation avant changement (passation 1) et de la passation après le changement (passation 2) sur l'échantillon commun aux deux passations ( $n=15$ sujets). 
Figure 3. Localisation des douleurs ou gênes perçues au cours des sept derniers jours

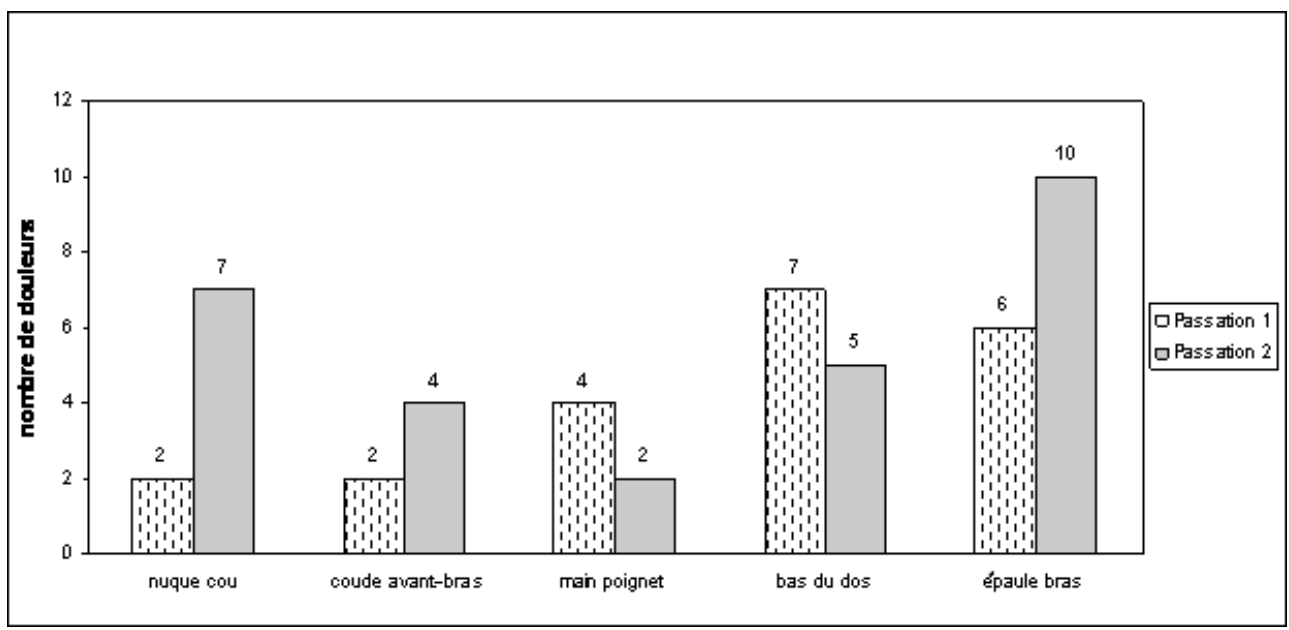

Comparaison des résultats issus de la passation avant changement (passation 1) et de la passation après le changement (passation 2) sur l'échantillon commun aux deux passations ( $n=15$ sujets).

L'analyse des facteurs de risques montre une diminution globale des contraintes biomécaniques perçues (cf. figure 4). Après le changement, le nombre d'opérateurs signalant devoir "travailler les bras en l'air ", "fléchir les coudes » ou avoir à « répéter les mêmes actions deux à quatre fois par minute » est plus faible qu'avant le changement. À l'inverse, les contraintes organisationnelles impactant le rythme de travail sont plus élevées lors de la seconde passation que lors de la première (cf. figure 5). Cette augmentation est principalement liée à l'implantation des dispositifs techniques, en particulier à la machine de tri du courrier dont dépendent directement les facteurs (déplacement automatique d'un produit, cadence automatique d'une machine, autres contraintes techniques). Les contraintes liées «aux autres » (collègues ou hiérarchie) apparaissent quand à elles moindres, ce qui peut laisser supposer que le collectif est une ressource pour l'activité de chacun.

Figure 4. Perception des contraintes biomécaniques : nombre de sujets déclarant devoir adopter les positions suivantes plus de deux heures/jour

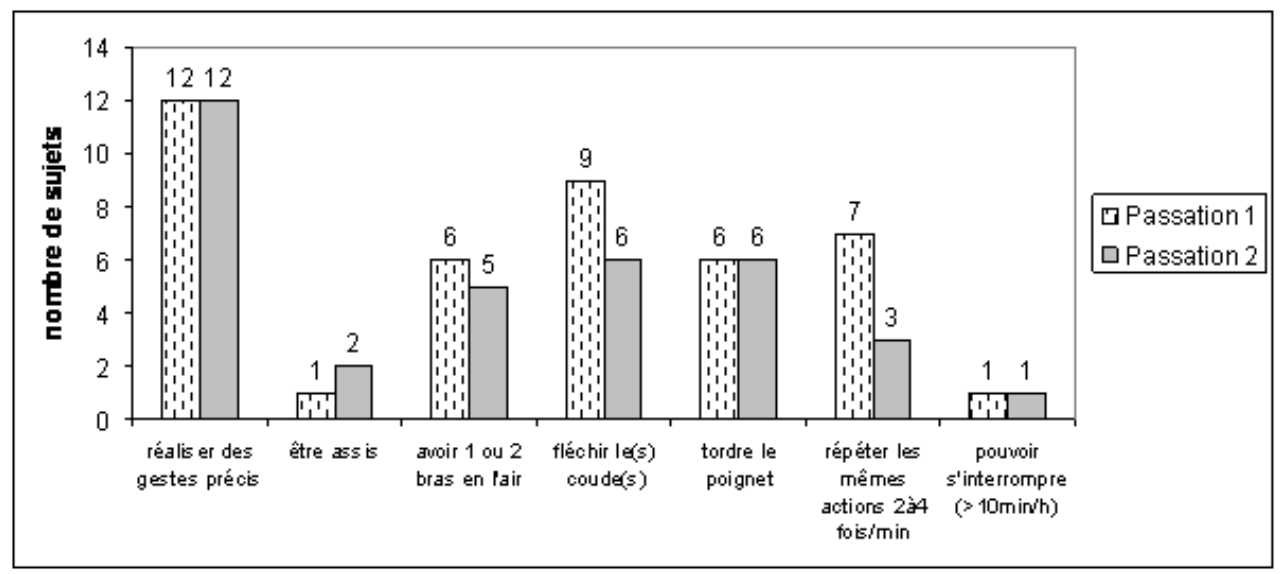

Comparaison des résultats issus de la passation avant changement (passation 1) et de la passation après le changement (passation 2) sur l'échantillon commun aux deux passations ( $n=15$ sujets). 
Figure 5. Perception des contraintes organisationnelles : nombre de sujets déclarant que leur rythme de travail est imposé par les contraintes organisationnelles suivantes

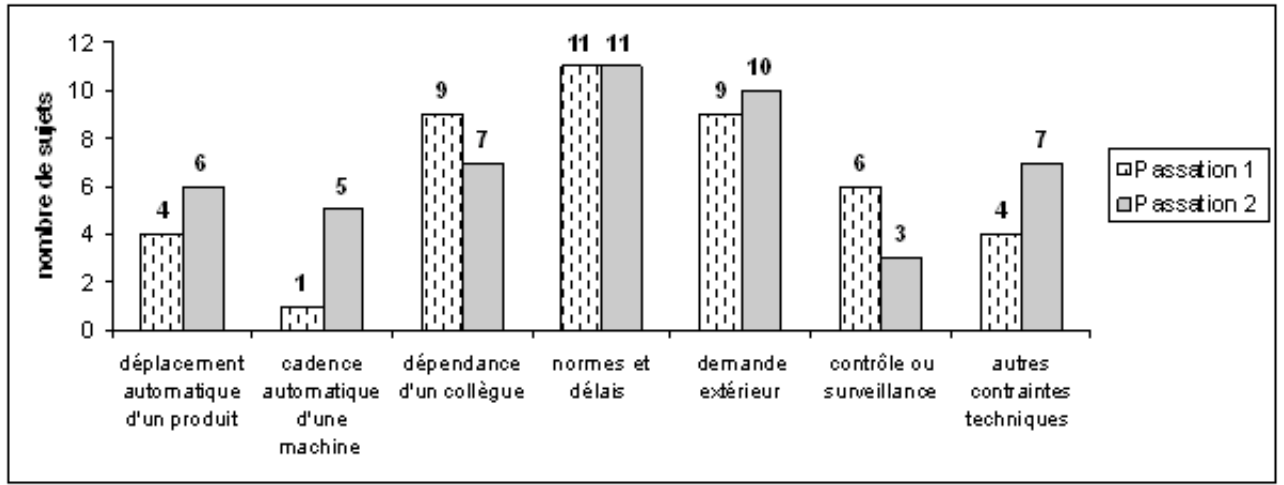

Comparaison des résultats issus de la passation avant changement (passation 1) et de la passation après le changement (passation 2) sur l'échantillon commun aux deux passations ( $n=15$ sujets).

Avec le changement, les contraintes de travail perçues par les opérateurs sont donc modifiées, et en particulier, les contraintes organisationnelles apparaissent plus importantes. En parallèle, les douleurs ressenties ont évolué quantitativement et qualitativement. Ainsi, même si les contraintes biomécaniques perçues ont diminué (peut-être avec l'introduction de nouveaux dispositifs comme le nouveau casier de tri), la santé semble avoir été affectée. Cette analyse quantitative invite à « donner un sens aux données» en articulant les résultats avec l'analyse qualitative de l'activité durant le changement (Volkoff, 2005).

\subsubsection{Analyse qualitative du vécu du changement}

Le changement, au cours duquel " tout a été modifié en même temps ", " par la force », a été vécu comme un épisode "stressant» ("un stress phénoménal ", "un climat de tension générale ») à tous les niveaux hiérarchiques ${ }^{2}$ : «Un changement, c'est franchir une étape, c'est fatigant pour tout le monde... En plus celui-ci a eu lieu en période très chargée... » En effet, le mois de janvier est la période la plus lourde pour les facteurs: aux aléas climatiques s'ajoutent les traditionnels envois de cartes de vœux et de cadeaux qui augmentent considérablement leur charge de travail. Dans ce contexte, l'apprentissage des nouvelles parties de tournées ainsi que l'«accoutumance nécessaire aux nouveaux lieux et matériels» ont généré des dépassements horaires importants : «Faire des heures supplémentaires tous les jours, ça met mal à l'aise » alors on "regarde l'heure tout le temps et [on] se dit plus vite, plus vite, plus vite. » La médecine de prévention professionnelle souligne, à la suite d'une visite de l'établissement un mois après le changement, " le stress et la fatigue des agents dans les suites de la réorganisation, en lien avec la phase d'apprentissage et les dépassements horaires quotidiens, d'une amplitude significative.» Très vite, une majorité d'agents ont préféré prendre leur poste plus tôt (jusqu'à trente minutes avant l'heure officielle) pour s'avancer dans le tri et ne pas trop décaler la journée: «Moi je rentrais chez moi, je n'avais presque même pas faim ; juste envie de m'allonger ». Au-delà de cet "état de fatigue à peu près général ", de "cette difficulté à récupérer, même le lendemain ", les facteurs évoquent fréquemment un "état de bien-être qu'[ils] avaient avant, entre [eux] » et qui s'est aujourd'hui "perdu »; en ayant laissé place à une "sensation de ne plus être bien dans sa peau ", de "ne plus avoir le cœ ur à venir au travail » ou d'y "venir à reculons. » 
26 Au fil des différents entretiens, plusieurs éléments ont été évoqués pour expliquer ce « mal-être » et cet "état de fatigue ». Ils peuvent être liés à la conduite du changement ou à certains de ses effets :

- le fait que le changement ait été introduit « tout en même temps » et non " par étapes »,

- l'augmentation du temps de distribution: « une demi-heure à quarante minutes de distribution en plus, c'est crevant ",

- les nouveaux moyens de locomotion : «Travailler en scooter, même si c'est moins physique pour les jambes, ça demande plus d'effort car ils sont lourds et peu manœuvrables ",

- la nouvelle organisation du travail : «On préfere manger à des heures correctes et venir une demiheure plus tôt le matin.",

- le nouveau casier de tri, dont les cases, plus nombreuses, plus étroites (1 ou $2 \mathrm{~cm}$ ) et orientées verticalement demande « une plus grande attention ». Désormais, il faut être « hyper concentré », « appliqué tout le temps » et "c'est usant ».

En filigrane de ces explications, la dimension collective est toujours présente dans le discours des facteurs : «On ne se voit plus », « on ne s'entend plus », « on ne se parle plus », « il n'y a plus de solidarité ", "plus d'ambiance ", " avant on travaillait autrement, là c'est l'usine, ce n'est plus convivial. » Lors du premier entretien collectif, les aspects collectifs du travail revenaient systématiquement au centre des échanges, en particulier lorsque les chercheurs abordaient avec les professionnels les liens entre les changements apportés et les effets sur leur santé. En effet, l'analyse de l'activité révèle que ce changement a perturbé les régulations collectives et déstabilisé le collectif de travail.

\subsection{Le changement et l'activité collective}

\subsubsection{Les formes du travail collectif et leurs évolutions avec le changement}

Dans les discours, le métier de facteur est souvent présenté comme un «métier autonome ". "Facteur, c'est un boulot individualiste ». "L'indépendance » fait même, pour certains, " tout l'intérêt du métier.» Mais l'emploi de ces qualificatifs, qui sont d'ailleurs loin d'être partagés par tous, se fait plus spécifiquement en référence à la distribution. À l'inverse « la préparation et le tri » sont généralement reconnus comme des phases de "travail en équipe » durant lesquelles "il peut y avoir de l'entraide ». Les résultats des observations et des entretiens montrent que les formes de réalisation de ce travail collectif ont été impactées par le changement apporté. Les exemples développés dans les paragraphes suivants illustrent ces modifications.

Le « tri général » communément appelé «TG3 » est la première étape de tri, durant laquelle l'équipe de facteurs répartit les plis en fonction des tournées. Elle se réalise sur des casiers à cases horizontales sur lesquels chaque case correspond à une tournée. Avant le changement, durant le tri général, la tâche prescrite était commune à tous les facteurs : elle se présentait comme un nombre de sacs à traiter chaque matin par l'ensemble de l'équipe. Le travail collectif se caractérisait par la présence de plusieurs opérateurs dans un même lieu, accomplissant ensemble la même opération (prendre des plis et les distribuer dans les cases des casiers du TG3) sur le même objet (l'ensemble des plis arrivés le matin) avec un but proximal commun (séparer ces plis par «quartiers» i.e. par tournées). Il s'agissait donc d'une coopération, «impliquant un partage du travail en fonction des connaissances et compétences, d'une certaine main-d'œuvre et force physique présente, de l'état de santé des uns et des autres et des exigences immédiates du 
travail » (De La Garza et Weill Fassina, 2000, p. 228). En effet, des régulations collectives ont été observées durant cette première étape de tri. Par exemple, avant le changement, les plis étaient, de façon "officieuse », séparés en deux zones, "les X et les Y " (en référence au code postal). Les facteurs s'étaient alors «spécialisés pour les $X$ ou pour les $Y$ » en fonction de leur tournée, ce qui leur « permettait de trier sur 15 à 20 cases, au lieu de 25 à 30. [Ainsi], il était possible de ne trier que sur les cases du bas ou devant soi, à sa hauteur ; l'avantage [étant] une fatigue moindre, un meilleur rendement et avec moins d'erreur, puisque les deux villes ont plusieurs noms de rue en commun ».

Par ailleurs, avant le changement, plusieurs situations d'entraide ont été observées. Durant les approvisionnements, par exemple, (lors du TG3 ou du TG4) il était fréquent de voir un facteur rapporter du courrier pour un ou plusieurs collègues voisins. Il en est de même lorsqu'il s'agissait d'aller chercher «les rebus » [i.e. lettres mal classées lors du TG3, qui se retrouvent donc sur de mauvaises "positions" pour le TG4]. Les facteurs les ramènent normalement en fin de tri sur les casiers du TG3. Mais « si tu vois que tu es avancé dans ton travail, tu peux rapporter directement aux collègues et même, si il y a pas trop de courrier, tu peux lui replacer, au bon endroit dans ses cases. " Avant le changement, il "était aussi possible de venir dépanner un gars dans la m... À l'aide de la fiche descriptive de la tournée, on pouvait lui trier une case ou deux. » Un autre exemple peut être le système d'entraide qui se mettait en place lors des réorganisations (permutation de tournées entre facteurs) : des informations et des fiches étaient échangées pour «faire face à l'incapacité des opérateurs » devant leur nouvelle tournée (De la Garza et Weill-Fassina, 2000, p. 228). « Car être titulaire d'une tournée, c'est beaucoup de connaissances": savoir exactement la localisation des boîtes aux lettres, anticiper les astuces des rues « tricotées ${ }^{3}$ ", les raccourcis, mais aussi connaître les clients et leurs habitudes, les jours d'ouverture des entreprises, « les indices qui, par exemple, signalent que la personne est absente et qu'il est inutile d'insister pour remettre un recommandé ». Certains consignent ces informations sur la fiche qui récapitule dans l'ordre les points de distribution de la tournée et peuvent donc les transmettre plus facilement à leurs collègues. De plus en plus, ces fiches sont «informatisées ». "On les garde en mémoire chez soi sur son ordinateur perso » et " on s'échange des disquettes » lors des réorganisations.

31 Ainsi, pour les facteurs, même si l'entraide concerne aussi la distribution, les régulations collectives se développent principalement durant les travaux de tri. Les changements techniques et organisationnels qui visent à diminuer ces phases de tri au profit des tâches de distribution, engendrent donc nécessairement une diminution du travail collectif. Par ailleurs, " pour suivre les cadences lors de cette phase du TG3, [les organisateurs] souhaitent que les trieurs ne se déplacent plus, quitte à avoir un trieur en moins, chargé de l'approvisionnement. » De ce fait, chaque facteur trie désormais le courrier qui lui est apporté. L'objet n'est donc plus commun et cette étape du TG3 n'est plus une « coopération » puisque les facteurs ne travaillent plus ensemble sur "un unique tas de courrier». Ils n'ont dès lors plus la possibilité de gérer ensemble «les interférences », de mettre en œuvre des régulations assurant "un partage du travail en fonction des connaissances et compétences » de chacun (De la Garza et Weill-Fassina, 2000). La régulation collective qui consistait à diviser en deux zones les « $\mathrm{X}$ et $\mathrm{Y}$ » au TG3 n'est de ce fait plus possible à mettre en œuvre. En outre, cette « chasse aux déplacements », associée au fait que, dans ce contexte de dépassement horaire, les facteurs ne prenaient pas de pauses, a supprimé « les temps improductifs» de discussions. Or, on sait que ces temps ne sont pas «des temps morts mais des temps forts » qui permettent non seulement la récupération mais 
aussi l'anticipation d'événements et les régulations collectives tels les aménagements entre collègues et les entraides décrites précédemment (Bourgeois et coll., 2006, p. 186). Les modifications techniques viennent aussi perturber ces « habitudes d'entraide ». Avec le nouveau casier, par exemple, il n'est plus possible de venir trier une ou deux cases pour un collègue trop chargé: "Avec le nouveau casier, on va d'un bout à l'autre progressivement, ce n'est plus possible de filer un coup de main sur une case... On peut ramener la caissette de TG4 à quelqu'un, mais cela n'est pas grand-chose. » " Aujourd'hui un gars qui est dans la m..., il reste dans la m...» Ainsi, le changement a modifié les formes du travail collectif: les phases de coopération, durant lesquelles s'étaient développées des stratégies de régulation et d'entraide, ont été diminuées au profit des phases de collaboration. De nouvelles modalités de régulations collectives doivent donc être développées au sein de l'équipe, ce qui nécessite du temps d'échanges et de débats entre collègues. Nos résultats révèlent qu'à l'inverse, durant ces périodes de changement, les réunions d'équipes ont été supprimées et que les temps de pauses et les possibilités de négociations informels entre collègues ont été fortement réduits.

\subsubsection{Le collectif de travail déstabilisé}

Par ailleurs, les résultats montrent que le changement effectué a engendré la remise en question des règles de métier auparavant établies sur le site. En voici un exemple. Avant le changement, sur le site de notre analyse, le TG3 était sensé se terminer à heure fixe. En réalité, la tâche effective que nous avons observée se présentait plutôt comme un nombre de sacs à traiter chaque matin par l'ensemble de l'équipe, et la fin du TG3 dépendait de la quantité de courrier. Cette redéfinition de la tâche était sous-tendue par un principe implicitement admis, "une conception du métier», qui voulait que l'« on ne laisse pas de papiers derrière soi »: "ça ne se fait pas ». On peut ici parler de « règles de métier ». Dans l'ancien centre de distribution,

«il exist[ait], entre l'organisation du travail et le sujet lui-même, un travail de réorganisation de la tâche par les collectifs professionnels, une re-création de l'organisation du travail par le travail d'organisation du travail collectif » (Clot, 2000, p. 273-274).

Avec le changement, les horaires ont été modifiés (arrêt du TG3 prévu à $7 \mathrm{~h} 30$ ) et «la machine n'a pas fonctionné à plein régime du premier coup » : durant la première semaine, la proportion de courrier trié mécaniquement était estimée entre 30 et $40 \%$ (contre $60 \%$ prévu), car "le nombre de " rejets » était important ». Durant cette période, les règles de métier ont été rediscutées (cf. tableau 2). 


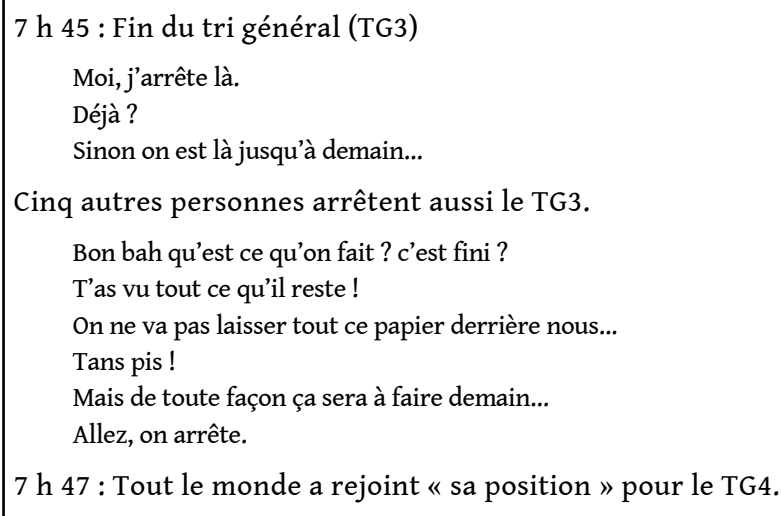

Ce débat s'est renouvelé les trois premiers jours. À la fin de notre intervention, le TG3 s'arrêtait à $7 \mathrm{~h} 30$ pile. D'autres sujets, comme

« embaucher avant l'heure ou non » ou bien comme «sortir les réexpéditions avant de partir en distribution comme le stipule le nouveau règlement ou passer outre » ont suscité des polémiques similaires. "Ces tiraillements entre variantes qui s'affrontent sont [...] souvent le signe que l'on cherche à stabiliser un genre » (Clot, 2000, p. 277).

Ainsi, le changement, en bouleversant certaines règles de métier, a déstabilisé le collectif de travail. Des débats comme celui présenté sont nécessaires pour que les opérateurs puissent se construire de nouvelles règles collectives et faire évoluer le métier de façon concomitante aux transformations organisationnelles et techniques. Mais cette fois aussi, ces disputes professionnelles autour de la définition « d'un travail bien fait » nécessitent que les controverses et les désaccords puissent être exprimés et débattus.

\section{Discussion}

Ces résultats présentent plusieurs limites. Les principales sont liées aux modalités de passation du questionnaire. Celle-ci repose en effet sur le volontariat, ce qui n'assure pas la représentativité de l'échantillon (Volkoff, 2005). Par ailleurs, dans ce contexte de changement, l'appropriation de la nouvelle situation est continue et la construction des gestes professionnels s'étend sur une durée bien supérieure à celle de notre étude. Les moments de réalisation de la passation sont donc primordiaux. Or, ils ont été contraints par la durée de l'intervention. Une seule passation a eu lieu avant le changement, et ceci à quelques jours de la mise en œuvre de ce dernier. Tandis que la passation après le changement a eu lieu quatre mois après que les transformations aient été effectuées. Les moments optimaux de réalisation de ces passations sont difficiles à évaluer. Après le changement,

«il ne faut pas évaluer trop tôt les transformations réalisées, et [d'un autre coté], plus le temps passe, plus l'évaluation comporte le risque de mettre en avant des effets qui ne sont pas [ceux du changement observé]» (Coutarel, 2004, p. 382; Coutarel et coll., 2009).

Le moment de la seconde passation du questionnaire nous est apparu intéressant: il correspondait au moment où les stratégies opératoires commençaient à se stabiliser. Mais 
d'autres passations ultérieures auraient pu venir enrichir les résultats et révéler des douleurs se manifestant sur du plus long terme. Le moment de la première passation nous semble par contre beaucoup trop proche du changement: nous pouvons penser que le changement imminent a influencé la perception des travailleurs sur les conditions de réalisation du travail et de ses effets. Il aurait été fortement préférable de pouvoir mener plusieurs passations bien avant la mise en œuvre du changement. Cette limite concerne aussi l'analyse de l'activité: on peut regretter de n'avoir pas pu mener davantage d'observations et d'entretiens avant l'implantation des nouveaux artéfacts et de la nouvelle organisation.

Malgré ces limites, les résultats montrent que cette période de changement fut une période d'instabilité, au cours de laquelle les opérateurs ont été fragilisés. Elle a en effet été vécue comme « une épreuve » « difficile » et « fatigante ». Les résultats quantitatifs du questionnaire confirment un impact sur la santé des opérateurs, et plus particulièrement sur la santé des opérateurs déjà fragilisés avant la mise en œuvre du changement. Mais, au-delà d'une évolution des contraintes physiques et organisationnelles de travail, la mise en œuvre du changement a aussi indirectement impacté l'activité collective : les formes de réalisation du travail collectif développées avant le changement sur le site (qui visaient entre autres des régulations collectives et des entraides) ont été altérées et les règles de métier ont dû être rediscutées au regard des évolutions techniques et organisationnelles. Or, ces discussions ne peuvent avoir lieu que si des espaces sont laissés aux débats de métier.

«Lorsque les débats de métier sont insuffisamment possibles, les règles de métier peuvent se retrouver en retard de phase par rapport au développement des moyens de production » (Daniellou, Simard et Boissière, 2009).

Ceci génère des risques à la fois pour la santé des opérateurs et pour la performance globale du système.

Notre intervention sur le site de production a favorisé ces débats de métier. Le questionnaire et les entretiens individuels avec les facteurs et l'encadrement ont permis de positionner «le travail» au cœur des échanges et de construire une nouvelle représentation de la santé, pas uniquement conditionnée par l'environnement technique. Les entretiens collectifs ont amené les opérateurs à verbaliser leur propre activité, à expliquer les exigences des situations de travail et à dévoiler leurs « combines » de métier pour y faire face.

«Ce processus d'extériorisation [leur] permet de comprendre les causes qui affectent leur comportement», causes qui sont bien souvent implicites (Falzon, 2005a).

41 Mais aussi, puisque ces entretiens ont été réalisés en groupe, ils ont encouragé le partage d'expérience, le transfert de connaissances et même ont pu produire de nouvelles connaissances, individuelles ou collectives. Ils ont donc contribué à élargir les gammes des gestes possibles et à rediscuter le rapport du collectif au "métier ». On peut soulever à ce propos que deux opérateurs nous ont indiqué avoir changé de stratégie ou «avoir testé un peu différemment» peu de temps après le premier entretien collectif. Nous avons, par ailleurs, mis en évidence le rôle joué par le collectif dans le développement des gestes et des instruments et montré que l'appropriation d'une stratégie opératoire s'articule avec le développement du collectif de travail (Cuvelier et Caroly, 2009).

42 Par la suite, deux réunions de restitution ont été organisées avec le management de proximité et l'ensemble de l'équipe, en présence du médecin du travail. Au cours de ces 
échanges, il a été convenu que, dans l'objectif de préserver et de construire la santé, il était déconseillé d'interdire certaines stratégies opératoires. Bien au contraire, la diversité des gestes a pu être encouragée dans le but de permettre à chacun d'employer la stratégie la plus adaptée à chaque moment du travail, dans un compromis entre les contraintes et les objectifs fixés par l'entreprise, mais aussi fixés par chacun individuellement et par le collectif de travail. Notre intervention a aussi démontré que l'organisation de réunions collectives de ce type (i.e. visant à mettre en débat, sans jugement de valeur, les gestes de métier) est une méthode qui permet aux individus et aux collectifs d'élargir leur gamme de gestes disponibles, ce qui est favorable à la santé. Ceci tout particulièrement en périodes de changement durant lesquelles les gestes de métier sont remis en cause et à reconstruire.

Au niveau de la direction de l'entreprise, les résultats de l'intervention ont été saisis par différents acteurs (direction, responsable de production, concepteurs du casier, médecine $\mathrm{du}$ travail) pour réinterroger la conduite du changement. Deux réunions de restitution ont ainsi été organisées successivement aux niveaux régional puis national. Elles ont été l'occasion d'envisager que, dans ce contexte de changements permanents, la prévention des TMS ne doit pas viser la mise en place de modèles cibles mais doit plutôt être abordée sous l'angle d'un processus dynamique dans la façon de conduire les changements (Daniellou, 2005). Car, comme constaté dans d'autres études, les résultats de cette intervention ont montré aux responsables que les problèmes de santé n'étaient pas tant liés aux conséquences des changements en eux-mêmes (et en particulier aux nouveaux dispositifs techniques) qu'à

« la façon de les mener en s'affranchissant de l'indispensable travail d'organisation que réalisent les salariés pour réussir à travailler correctement dans la durée » (Clot, 2010, p. 103).

C'est en voulant éviter les controverses et les « disputes » collectives sur la définition d'un travail «bien fait » que la santé au travail est mise à mal. Ces résultats et les échanges qu'ils ont générés ont ainsi permis de revaloriser les espaces et les temps dédiés aux « débats de métier » durant ces périodes de changements.

\section{Conclusion}

Les résultats de cette intervention mettent en évidence l'évolution des contraintes liées à la mise en œuvre d'un changement organisationnel et technique majeur et leurs impacts sur la santé. Ils montrent combien l'articulation entre les changements techniques et les évolutions du métier portées par les collectifs est un enjeu majeur de la santé au travail. Il apparait donc primordial de veiller à préserver ces collectifs professionnels durant les périodes de mutation, en conservant des espaces dédiés aux « débats de métier ».

Au-delà, cette intervention illustre comment les résultats et les méthodes déployées par l'approche ergonomique peuvent être saisis par les acteurs de différents niveaux hiérarchiques de l'entreprise pour requestionner leur façon de conduire les changements. Ils viennent conforter les résultats d'autres études (Dugué, Chassaing, Coutarel et Daniellou, 2010) qui montrent qu'au-delà des recommandations ergonomiques, la démarche ergonomique propose des méthodes d'accompagnement du changement que les acteurs de l'entreprise peuvent s'approprier et réutiliser à plus long terme. 
Nous tenons à remercier l'équipe du centre de tri et de distribution du courrier qui nous a accueillies, et tout particulièrement le Dr Véronique Arnaudo, médecin de prévention professionnelle avec qui nous avons étroitement collaboré durant cette étude.

\section{BIBLIOGRAPHIE}

Aptel, M., Cail, F. et Aublet-Cuvelier, A. (2009). Les troubles musculosquelettiques du membre supérieur (TMS-MS) : guide pour les préventeurs : ED 957- INRS.

Aptel, M. et Hubault, F. (2005). La prévention durable des TMS : des expériences et des points de vue. Les conditions d'une prévention durable des TMS (p. 16-20). Études et documents. Lyon : ANACT.

Aptel, M. et Gaudez, C. (2005). Plausibilité biologique du lien entre les TMS-MS et le stress. Actes du $1^{\text {er }}$ Congrès francophone sur les TMS du membre supérieur. Nancy.

Assunçao, A. (1998). De la déficience à la gestion collective du travail : les troubles musculosquelettiques dans la restauration collective. Thèse de doctorat en ergonomie, Paris 5 : École pratique de hautes études.

Barthe, B. (2000). Travailler la nuit au sein d'un collectif : quels bénéfices ? In Benchekroun, T.H. et Weill-Fassina, A. (Coo.), Le travail collectif. Perspectives actuelles en ergonomie. (p. 235-255). Toulouse : Octarès Éditions.

Béguin, P. (2004). L'ergonome, acteur de la conception. In P. Falzon (Ed.), Ergonomie (p. 375-390). Paris : PUF.

Bellemare, M., Marier, M. et Allard, D. (2001). Le journal de bord : un outil pour l'intervention et la recherche. Actes du Congrès SELF ACE 2001. Les transformations du travail, enjeux pour l'ergonomie. Vol. 3, p. 58-62.

Bernoux, P. (2004). Sociologie du changement dans les entreprises et les organisations. Paris : Éditions du Seuil.

Bobillier Chaumon, M.E. et Dubois, M. (2007). Les mutations du travail face aux défis technologiques : Quelles incidences pour la santé. Pistes, 9, 2. www.pistes.uqam.ca

Bourgeois, F., Lemarchand, C., Hubault, F., Brun, C., Polin, A., Faucheux, J.M., Douillet, P. et Albert, E. (2006). Troubles musculosquelettiques et travail. Quand la santé interroge l'organisation. Lyon : ANACT.

Caroly, S. (2010). L'activité collective et la réélaboration des règles : des enjeux pour la santé au travail. Habilitation à diriger des recherches - Mention ergonomie, Université Victor Segalen, Bordeaux 2.

Caroly, S. et Clot, Y. (2004). Du travail collectif au collectif de travail : développer des stratégies d'expérience. Formation Emploi, $\mathrm{n}^{\circ}$ 88, p. 43-55.

Clot, Y. (2000). La fonction psychologique du collectif. In Benchekroun, T.H. et Weill-Fassina, A. (Coo.), Le travail collectif. Perspectives actuelles en ergonomie (p. 272-286). Toulouse : Octarès Éditions. 
Clot,Y., Scheller, L., Caroly, S., Millanvoye, M. et Volkoff, S. (2000). Les chemins de l'aptitude. Synthèse d'une étude menée dans deux bureaux de poste. Rapport de recherche des laboratoires CNAM et CREAPT.

Clot, Y. (2004). La fonction psychologique du travail. $4^{\mathrm{e}}$ édition corrigée. Paris : PUF.

Clot, Y. et Fernandez, G. (2005). Analyse psychologique du mouvement : apport à la compréhension des TMS. @ctivités, vol. 2, n², p. 68-78.

Clot, Y. (2005). Les TMS : hyper-sollicitation ou hypo-sollicitation? Actes du $1^{\text {er }}$ Congrès francophone sur les TMS du membre supérieur. Nancy.

Clot, Y. (2010). Le travail à cœur. Pour en finir avec les risques psychosociaux : La Découverte, coll. Cahiers libres.

Coutarel, F. (2004). La prévention des troubles musculo-squelettiques en conception : quelles marges de manœuvre pour le déploiement de l'activité ? Thèse de doctorat en ergonomie, Université Victor Segalen Bordeaux 2. Collection Thèses et mémoires du Laboratoire d'ergonomie des systèmes complexes.

Coutarel, F., Vézina, N., Berthelette, D., Aublet-Cuvelier, A., Descatha, A., Chassaing, K. et coll. (2009). Orientations pour l'évaluation des interventions visant la prévention des troubles musculo-squelettiques liés au travail. Pistes, 11(2), www.pistes.uqam.ca/v11n2/pdf/v11n2a1.pdf

Cuvelier, L. et Caroly, S. (2009). Appropriation d'une stratégie opératoire : un enjeu du collectif de travail. Activités, 6(2), 61-82, www.activites.org/v66n62/v66n62.pdf.

Cru, D. (1988). Collectif et travail de métier. In C. Dejours (Ed.), Plaisir et souffrance dans le travail. (p. 43-49). Paris : Édition de l'AOCIP.

Cru, D. (1995). Règle de métier. Langue de métier. Diplôme d'ergonomie. Paris : École pratique des hautes études.

Daniellou, F. (2004). L'ergonomie dans la conduite de projets de systèmes de travail. In P. Falzon (Ed.), Ergonomie (p. 359-373). Paris : PUF.

Daniellou, F. (2005). TMS et modèles d'organisation du travail et de la production. Actes du $1^{\mathrm{er}}$ Congrès francophone sur les TMS du membre supérieur. Nancy.

Daniellou, F., Simard, M. et Boissières, Y. (2009). Facteurs humains et organisationnels de la sécurité industrielle : un état de l'art. Toulouse : FonCSI.

Daniellou, F., Caroly, S., Coutarel, F., Escriva, E., Roquelaure, Y., Schweitzer, J.M. (2008). La prévention durable des TMS : Quels freins? Quels leviers d'action? Rapport final d'étude pour la Direction générale du travail.

Darses, F. et de Montmollin, M. (2006). L'ergonomie. Paris : Éditions La Découverte.

Davezies, P. (2005). La santé au travail, une construction collective. Santé et travail, $n^{\circ} 52$, p. 24-28.

Dessors, D., Dejours, C. et Molinier, P. (1994). Pour comprendre la résistance au changement, Documents pour le médecin du travail, $\mathrm{n}^{\circ} 58, \mathrm{p} .112-117$, Ed. INRS et ministère du Travail.

De la Garza, C. et Weill-Fassina, A. (2000). Régulations horizontales et verticales du risque. In Benchekroun, T.H. et Weill-Fassina, A. (Coo.), Le travail collectif. Perspectives actuelles en ergonomie. (p. 217-224). Toulouse : Octarès Éditions. 
Dubar, C., Demazière, D., Guardiola, A. et Mercier, D. (2001) Identités professionnelles, organisation du travail et performances : Le cas des facteurs de La Poste. Rapport final pour le ministère de la Recherche et la Mission Recherche de La Poste.

Dugué, B., Chassaing, K., Coutarel, F. et Daniellou, F. (2010). L'ergonome peut-il contribuer à créer des systèmes adaptatifs et résilients 5 ans après la conception d'une ligne de découpe, le retour dans un abattoir de canards gras. $45^{\mathrm{e}}$ Congrès de la Société d'ergonomie de langue française (SELF), 13-15 septembre, Liège, Belgique.

Falzon, P. (1996). Des objectifs de l'ergonomie. In F. Daniellou (Ed.), L'ergonomie en quête de ses principes (p. 233-242). Toulouse, Octarès.

Falzon, P. (2005). Developing ergonomics, developing people. Proceedings of the $8^{\text {th }}$ South East Asian Ergonomics Society Conference (SEAES-IPS) International conference : Bridging the gap, May 23-25. Denpasar, Bali, Indonesia, 1-10.

Flageul-Caroly, S. (2001). Régulations individuelles et collectives de situations critiques dans un secteur de service : le guichet de La Poste. Paris : Thèse de doctorat d'ergonomie, EPHE.

Gaudart, C. et Pueyo, V. (2000). L'expérience dans les régulations individuelles et collectives de l'efficience. In Benchekroun, T.H. et Weill-Fassina, A. (Coo.), Le travail collectif. Perspectives actuelles en ergonomie. (p. 257-271). Toulouse : Octarès Éditions.

Gaudart, C. et Weill-Fassina, A. (1999). L'évolution des compétences au cours de la vie professionnelle : une approche ergonomique.

Guibert, S. (2009). L'évaluation ergonomique de la performance d'un changement organisationnel. Thèse de doctorat en ergonomie, CNAM, Paris.

Hoc, J.-M. (2001). Towards a cognitive approach to human-machine cooperation in dynamic situations. International Journal of Human-Computer Studies, 54(4), 509-540.

Hubault, F. (2005). Choisir un modèle du risque qui permet d'y répondre durablement. Les conditions d'une prévention durable des TMS (p. 21-26). Études et documents. Lyon : ANACT.

Lacoste, M. (1992). Apprentissage dans le travail et interaction. Langage et travail, $n^{\circ} 3$, p. 35-42.

Lasfargues, G., Roquelaure, Y., Fouquet, B. et Leclerc, A. (2003). Pathologie d'hypersollicitation périarticulaire des membres supérieurs. Troubles musculo-squelettiques en milieu de travail. Paris : Masson.

Lamonde, F. (2004). Les prescriptions des ergonomes. In P. Falzon (Ed.), Ergonomie (p. 391-404). Paris : PUF.

Landry, A. (2008). L'évaluation de l'intervention ergonomique : de la recherche évaluative à la proposition d'outils pour la pratique. Thèse de doctorat en ergonomie, Université Victor Ségalen Bordeaux 2, Bordeaux.

Laville, A. et Volkoff, S. (1993). Âge, santé, travail : le déclin et la construction. Actes du XXVIII ${ }^{\mathrm{e}}$ Congrès de la SELF, Genève, 22-24 septembre 1993.

Leplat, J. (1993) Ergonomie et activités collectives, in Six, F., Vaxevanoglou, X. (Eds), Les aspects collectifs du travail, Toulouse, Octarès.

Mollo, V. (2004). Usage des ressources, adaptation des savoirs et gestion de l'autonomie dans la décision thérapeutique. Thèse de doctorat en ergonomie, Cnam, Paris.

Mollo, V. et Falzon, P. (2004). Auto- and allo-confrontation as tools for reflective activities. Applied Ergonomics, 35(6), 531-540. 
Pavageau, P., Nascimento, A. et Falzon, P. (2007) Les risques d'exclusion dans un contexte de transformation organisationnelle. Pistes, 9 (2), octobre 2007. www.pistes.uqam.ca

Prunier-Poulmaire, S. et Gadbois, C. (2005). Quand le questionnaire s'impose à l'ergonome. In Volkoff, S. (Coo.), L'ergonomie et les chiffres de la santé au travail : ressources, tensions et pièges (p. 75-86). Toulouse : Octarès Éditions.

Pueyo, V. (1999). Régulation de l'efficience avec l'expérience : quelles questions pour la construction d'indicateurs de suivi de la production? Actes du XXXIV ${ }^{\mathrm{e}}$ Congrès de la SELF. Caen.

Roquelaure, Y., Mariel J., Fanello, S., Boissiere, J.C., Chiron, H., Dano, C., Bureau, D. et PenneauFontbonne, D. (2002). Active epidemiological surveillance of musculoskeletal disorders in a shoe factory. Occup Environ Med n 59, p. 452-458.

Roquelaure, Y., Malchaire, J., Cock, N., Martin, Y.H., Piette, A., Vergracht, S., Chiron, H. et Le Boulanger, M.A. (2001). Évaluation d'une méthode de quantification de l'activité gestuelle au cours de tâches répétitives de production de masse. Document pour le médecin du travail, $\mathrm{n}^{\circ} 86$, p. $167-176$.

Schmidt, K. (1991). Cooperative work : A conceptual framework : Introduction. In J. Rasmussen, B. Brehmer et J. Leplat (Eds), Distributed decision making : Cognitive models for cooperative work (p. 75-106). Chichester : John Wiley \& Sons.

Valot, C. (2001). Pour une ergonomie du changement dans les organisations. Actes du Congrès SELF ACE 2001. Les transformations du travail, enjeux pour l'ergonomie. vol. 3, p. 24-29.

Vézina, N. (2001). La pratique de l'ergonomie face aux TMS : ouverture à l'interdisciplinarité. Comptes rendus du Congrès SELF ACE 2001. Les transformations du travail, enjeux pour l'ergonomie. vol. 1, p. 45-60.

Volkoff, S. (2005). Des comptes à rendre : usages des analyses quantitatives en santé au travail pour l'ergonomie. In Volkoff, S. (Coo.), L'ergonomie et les chiffres de la santé au travail : ressources, tensions et pièges (p. 3-74). Toulouse : Octarès Éditions.

Weill-Fassina, A. et Benchekroun, T.H. (2000). Diversité des approches et objets d'analyse du travail collectif en ergonomie. In Benchekroun, T.H. et Weill-Fassina, A. (Coo.), Le travail collectif. Perspectives actuelles en ergonomie. (p. 217-224). Toulouse : Octarès Éditions.

Zanet, F., Hansez, I., Bossut, M., Vandenberghe, C. et De Keyser, V. (2004). Analyse du discours de travailleurs confrontés à des changements organisationnels : une perspective transactionnelle, Le travail humain 2004/3, volume 67, p. 257-281.

\section{NOTES}

1. Les termes de métier et les verbatim extraits des entretiens retranscrits sont en italique.

2. La médecine du travail stipule sur ce point que «les réorganisations successives [...] ont aggravé la charge de travail et le stress [des directeurs d'établissement]. [...]Depuis 2 ans, plusieurs épuisements professionnels ont été constatés ».

3. Les rues «tricotées » sont les rues pour lesquelles le facteur passe d'un coté de la rue à l'autre, en alternant donc les numéros pairs et impairs qui sont bien souvent décalés et donc ne se suivent plus. 


\section{RÉSUMÉS}

Cette intervention repose sur le suivi d'un changement organisationnel et technique opéré dans un centre de tri et de distribution du courrier. Elle vise à caractériser l'impact de ce changement sur l'activité et la santé des opérateurs. Plusieurs méthodes (observations, entretiens, diffusion d'un questionnaire) ont été mises en œuvre afin d'aborder (ou de comprendre) ces mutations et leurs conséquences. Les résultats révèlent que ce changement fut une période de fragilisation de la santé au travail, durant laquelle les formes de réalisation du travail collectif développées auparavant sur le site (qui visaient entre autres des régulations collectives et des entraides) ont été remises en cause. Ils ont été saisis par différents acteurs de l'entreprise pour réinterroger la conduite du changement. Les marges de manœuvre et les espaces laissés aux « débats de métier » apparaissent essentiels durant ces périodes de mutations.

This research is based on the analysis of an important organizational and technical change carried out in a sorting post office. It aims to characterize the impact of this change on the work activity and on occupational health. Several methods (observations, interviews and questionnaire) have been implemented to understand the consequences of this period on the health of operators and on the collective dimensions of work. The results show that this period was a period of instability during which the workers' health has been affected. They also show that this change has impacted the collective strategies previously developed by operators. Giving space for discussions and leeway to operators during these changes appears to be essential.

Esta intervención se basa en el seguimiento de un cambio organizacional y técnico que se llevó a cabo en un centro de clasificación y de distribución de correo. Ella desea caracterizar el impacto de este cambio sobre la actividad y la salud de los operadores. Varios métodos (observaciones, entrevistas, difusión de un cuestionario) fueron aplicados para comprender las mutaciones y sus consecuencias. Los resultados revelan que este cambio fue un periodo de fragilización de la salud en el trabajo, durante el cual las formas de realización del trabajo colectivo desarrolladas anteriormente (que aspiraban entre otras cosas a regulaciones colectivas y a la ayuda mutua) fueron puestas en duda. Esto fue utilizado por diferentes actores de la empresa para reinterrogar la conduccción del cambio. Los márgenes de maniobra y los espacios dejados a los «debates de oficio » aparecen como esenciales durante esos periodos de mutaciones.

\section{INDEX}

Palabras claves : cambio organizacional, trabajo colectivo, salud

Mots-clés : changement organisationnel, travail collectif, santé

Keywords : organizational change, collective work, health 


\section{AUTEURS}

\section{LUCIE CUVELIER}

Lucie.cuvelier@cnam.fr, Laboratoire PACTE, Université de Grenoble, Le patio, BP 47, 38040

Grenoble cedex 09, France

\section{SANDRINE CAROLY}

Sandrine.caroly@upmf-grenoble.fr, Cnam, Centre de recherche sur le travail et le développement (EA 4132), Équipe ergonomie, 41, rue Gay Lussac, 75005 Paris, France 